$10,12,18$

\title{
Поперечные пьезо- и пироэлектрический эфффекты в 2D-наноаллотропах нитрида бора, обусловленные риплообразованием
}

\author{
(С) Р.А. Браже, Д.А. Долгов
}

Ульяновский государственный технический университет, Ульяновск, Россия

E-mail: brazhe@ulstu.ru

Поступила в Редакцию 5 марта 2020 г.

В окончательной редакции 5 марта 2020 г.

Принята к публикации 17 марта 2020 г.

Показано, что образование рипплов (ряби) в структуре 2D-наноаллотропов нитрида бора приводит к возникновению в них поперечных пьезо- и пироэлектрического эффектов с зависящими от температуры коэффициентами. Рассчитана величина этих коэффициентов для различных 2D-наноаллотропов нитрида бора. Показано, что вследствие малой высоты рипплов поперечный пьезоэффект в рассматриваемых наноаллотропах на три порядка слабее продольного, в то время как пироэлектрический эффект вполне сравним с аналогичным эффектом во фторографанах и может найти практическое применение.

Ключевые слова: нитрид бора, 2D-наноаллотропы, пьезо- и пироэлектрический эффекты.

DOI: $10.21883 /$ FTT.2020.08.49612.046

\section{1. Введение}

Большой интерес для использования в наноэлектронике и наноэлектромеханических системах управления информацией представляют планарные и нанотубулярные кристаллические структуры, обладающие пьезоэлектрическими и пироэлектрическими свойствами. Они могут быть созданы на основе графена путем нарушения его центросимметричности перфорированием или допированием электроположительными и электроотрицательными атомами [1-5]. Однако, в связи с технологическими трудностями серийного изготовления таких структур и большими экономическими затратами, более перспективным представляется использование в указанных целях наноаллотропов нитрида бора (BN) и других $2 \mathrm{D}$-соединений типа $\mathrm{A}^{\mathrm{III}} \mathrm{B}^{\mathrm{V}}$, обладающих естественным пьезоэффектом [6-8]. Большой практический интерес представляет также использование пироэлектрических свойств гексогонального нитрида бора в составе нанокомпозитов [9].

В работе [7] были рассчитаны независимые компоненты тензора пьезомодулей, описывающих продольный пьезоэффект (в плоскости 2D-структуры) в различных 2D-наноаллотропах BN. Возникновение продольного пироэлектрического эффекта в таких наноструктурах возможно лишь при наличии градиентов температуры, что при их наномасштабных размерах практически нереализуемо.

Вместе с тем, как было показано в [5], нагревание 2D-кристаллических структур приводит к их гофрированию с образованием рипплов (от англ. ripple -- рябь). Изменение температуры вызывает изменение высоты рипплов и изменение длины диполей, образованных частичной полярностью связей между атомами В и $\mathrm{N}$.
Это, в свою очередь, вызывает изменение поляризованности структуры в направлении, перпендикулярном к ее поверхности, т. е.приводит к поперечному пироэлектрическому эффекту.

Целью данной работы является оценка численных значений компонент тензоров пьезоэлектрических и пироэлектрических коэффициентов, описывающие соответствующие явления вследствие рипплообразования в различных 2D-наноаллотропах нитрида бора.

\section{2. Исследуемые структуры, рипплы и методы расчета}

Исследуемые структуры показаны на рис. 1, а строение возникающих при их нагревании рипплов на рис. 2. Поляризованность структуры $P_{3}$ при некотором конечном значении температуры $T$

$$
P_{3}(t)=\frac{Z e h}{S} \frac{\partial u_{3}}{\partial x_{3}}=c_{333} \frac{\partial u_{3}}{\partial x_{3}},
$$

где $Z-$ число диполей в элементарной ячейке, $S-$ ее площадь, $e-$ элементарный заряд, $h-$ высота рипплов, $u_{3}$ - смещение частиц среды вдоль координаты $x_{3}$ (перпендикулярно поверхности структуры), $e_{333}-$ coответствующая компонента тензора пьезоэлектрических коэффициентов (в матричных обозначениях $e_{33}$ ).

При изменении температуры относительная деформация структуры вдоль оси $x_{3}$ :

$$
\frac{\partial u_{3}}{\partial x_{3}}=\alpha_{33} \Delta T,
$$

где $\alpha_{33}$ - компонента тензора теплового расширения в данном направлении (в векторных обозначениях $\alpha_{3}$ ). 

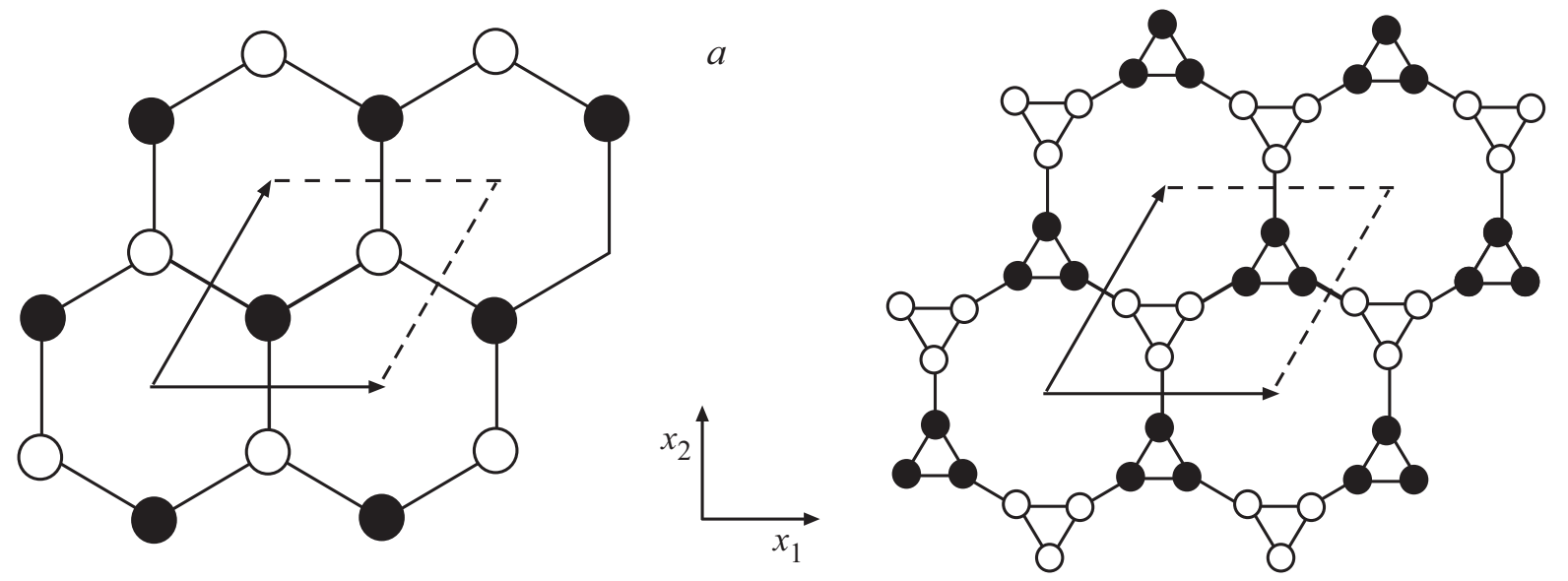

$b$
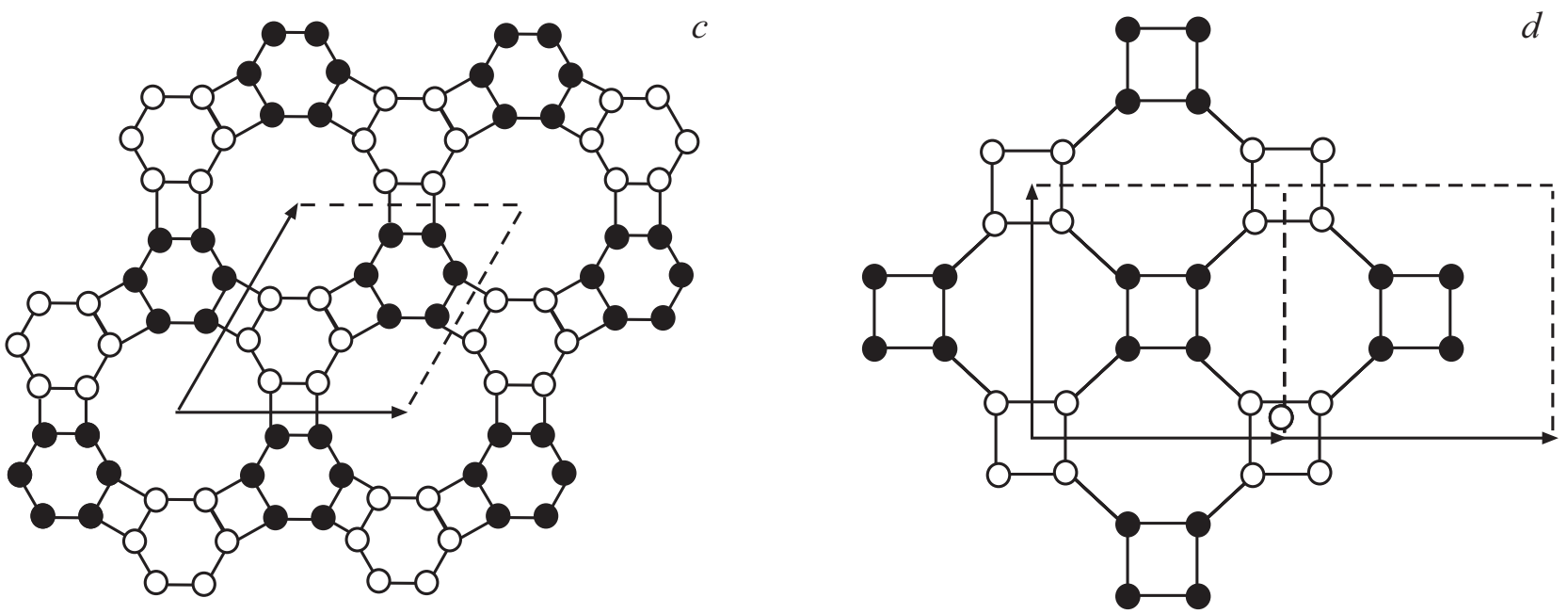

Рис. 1. Атомная структура с выделенной в ней элементарной ячейкой исследуемых 2D-наноаллотропов нитрида бора: $a-(\mathrm{BN})_{6}$, $b-(\mathrm{BN})_{63(12)}, c-(\mathrm{BN})_{664}, d-(\mathrm{BN})_{44}$.
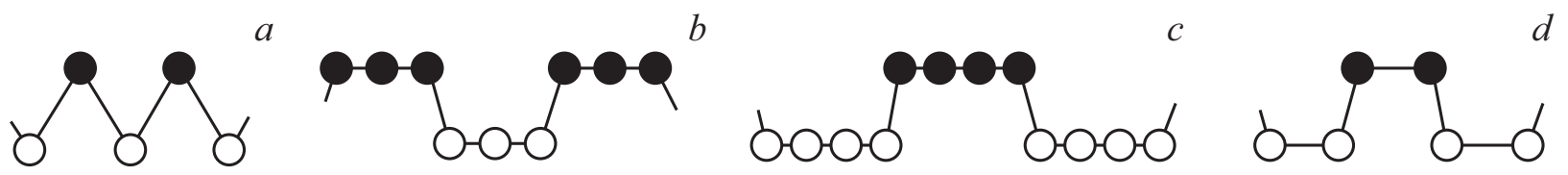

Рис. 2. Строение образующихся рипплов в $2 \mathrm{D}$-наноаллотропах, изображенных на рис. 1. (Вид вдоль структуры): $a-(\mathrm{BN})_{6}$, $b-(\mathrm{BN})_{63(12)}, c-(\mathrm{BN})_{664}, d-(\mathrm{BN})_{44}$.

Уравнение поперечного пироэлектрического эффекта имеет вид

$$
\Delta P_{3}=\gamma_{3} \Delta T,
$$

где $\gamma_{3}$ - пироэлектрический коэффициент. С учетом (1), (2) уравнение (3) можно переписать в виде

$$
\Delta P_{3}=e_{33} \alpha_{3} \Delta T .
$$

Высоту рипплов $h$ можно выразить через деформацию $\Delta x_{1}$ в плоскости атомной структуры, а саму величину $\Delta x_{1}$ через коэффициент теплового расширения (сжатия) $\alpha_{1}$ в плоскости этой структуры (см. рис. 3). Поскольку
$\Delta x_{1} \ll f_{0}$, где $f_{0}-$ характерный период структуры при температуре $T_{0}$, когда рипплов еще нет, то

$$
h \approx \sqrt{2 f_{0} \Delta x_{1}} .
$$

С другой стороны,

$$
\Delta x_{1}=\frac{\partial u_{1}}{\partial x_{1}} f_{0}=\left|\alpha_{1}\right| T f_{0} .
$$

Тогда

$$
h=f_{0} \sqrt{2\left|\alpha_{1}\right| T} .
$$




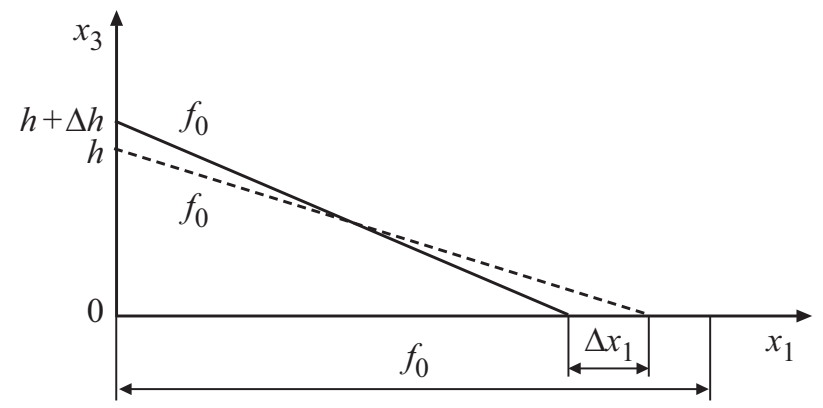

Рис. 3. Связь высоты рипплов с продольными деформациями структуры.

Отметим, что $\alpha_{3}$ и $\alpha_{1}$ имеют разные знаки, так как „вздутие“ 2D-структуры в направлении $x_{3}$ вызывается ее сжатием в продольном направлении $x_{1}$.

Изменение высоты рипплов с температурой

$$
\Delta h=\frac{\partial u_{3}}{\partial x_{3}} h=\alpha_{3} \Delta T h .
$$

С другой стороны

$$
\Delta h=\frac{\alpha_{1} f_{0} \Delta T}{\sqrt{2\left|\alpha_{1}\right| T}} .
$$

Откуда

$$
\alpha_{3}=\frac{1}{\sqrt{2} T}
$$

и не зависит от вида структуры.

Тогда, согласно (1)-(6),

$$
\begin{gathered}
e_{33}(T)=\frac{Z e}{S} f_{0} \sqrt{2\left|\alpha_{1}\right| T}, \\
\gamma_{3}(T)=\frac{\sqrt{2}}{2} \frac{e_{33}(T)}{T} .
\end{gathered}
$$

Входящая в выражения (7), (8) величина $\alpha_{1}$ может быть найдена по методике, изложенной в работе [5]. При температурах выше и ниже температуры Дебая $\theta_{D}$ (см. Приложение)

$$
\begin{gathered}
\alpha_{1}\left(T \gg \theta_{D}\right)=\frac{N k_{B}}{\alpha a_{\mathrm{BN}}^{2}} \gamma ; \\
\alpha_{1}\left(T \ll \theta_{D}\right)=14.5\left(\frac{T}{\theta_{D}}\right)_{1}^{2}\left(T \gg \theta_{D}\right),
\end{gathered}
$$

где $N$ - число атомов в элементарной ячейке, $k_{B}-$ постоянная Больцмана, $\alpha$ - константа центрального взаимодействия атомов, определяемая выражением $[10,11]$

$$
\alpha=\frac{2}{a_{\mathrm{BN}}^{2}} V_{2}\left[1-\frac{10}{3}\left(\frac{V_{1}}{V_{2}}\right)^{2}\right],
$$

$a_{\mathrm{BN}}-$ межатомное расстояние, $V_{2}$ и $V_{1}-$ соответственно ковалентная энергия $\sigma$-связи в $s p^{2}$-наноаллотропах

$$
V_{2}=3.26 \frac{h^{2}}{m a_{\mathrm{BN}}^{2}},
$$

a $V_{1}$ - энергия металлизации, которую в данном случае удобно вычислить, найдя $V_{2}$ по формуле (11) и используя затем выражение (10), куда подставим $\alpha$, вычисленные для рассматриваемых 2D-наноаллотропов в работе [12]. Заметим, что в выражении (10) мы для простоты пренебрегаем коэффициентом полярности $\sigma$-связи $[11]$, что оправдано, как показано нами в $[7,12]$, для квазиизотермических условий.

Входящая в (9) величины $\gamma$ является усредненным по фононному спектру параметром Грюнайзена, который можно найти как $[5,13]$ :

$$
\gamma=\frac{2 V_{2}}{\alpha a_{\mathrm{BN}}^{2}}\left[2-\frac{10}{3}\left(\frac{V_{1}}{V_{2}}\right)^{2}\right] .
$$

Подставляя (12) в (9) и используя выражения (7), (8), находим зависящие от температуры значения пьезоэлектрических и пироэлектрических констант для исследуемых 2D-наноаллотропов нитрида бора.

\section{3. Обсуждение результаты и выводы}

В табл. 1 представлены необходимые для расчетов геометрические параметры исследуемых 2D-наноаллотропов нитрида бора, а в табл. 2 результаты вычислений по формулам (7)-(12). Сравнивая их с результатами вычислений пьезоэлектрического коэффициента $e_{33}$ и пироэлектрического коэффициента $\gamma_{3}$, приведенными в работе [5] для фторографаноподобных 2D-наноаллотропов такой же симметрии: $(\mathrm{FG}-\mathrm{GH})_{6},(\mathrm{FG}-\mathrm{GH})_{63(12)}$, $(\mathrm{FG}-\mathrm{GH})_{664},(\mathrm{FG}-\mathrm{GH})_{44}$, можно заметить, что обусловленная рипплообразованием и характеризующая поперечный пьезоэффект пьезоконстанта $e_{33}$ в наноаллотропах $(\mathrm{BN})_{6},(\mathrm{BN})_{63(12)},(\mathrm{BN})_{664}$, и $(\mathrm{BN})_{44}$ в целом на три порядка меньше. Это связано, в первую очередь, с очень малой высотой рипплов по сравнению с длиной диполей во фторграфанах.

B то же время пироэлектрические коэффициенты $\gamma_{3}$ для 2D-наноаллотропов нитрида бора близки по вели-

Таблица 1. Геометрические параметры исследуемых 2D-наноаллотропов нитрида бора

\begin{tabular}{c|c|c|c|c}
\hline Параметр & $(\mathrm{BN})_{6}$ & $(\mathrm{BN})_{63(12)}$ & $(\mathrm{BN})_{664}$ & $(\mathrm{BN})_{44}$ \\
\hline$Z$ & 1 & 3 & 6 & 4 \\
$a_{\mathrm{BN}}, \AA$ & 1.45 & 1.6 & 1.44 & 1.38 \\
$f_{0}$ & $\frac{\sqrt{3}}{2} a_{\mathrm{BN}}$ & $\sqrt{3} a_{\mathrm{BN}}$ & $\frac{\sqrt{3}}{2} a_{\mathrm{BN}}$ & $\frac{\sqrt{2}}{2} a_{\mathrm{BN}}$ \\
$S$ & $\frac{3 \sqrt{3}}{2} a_{\mathrm{BN}}^{2}$ & $\frac{(2+\sqrt{3})^{2}}{2} a_{\mathrm{BN}}^{2}$ & $\frac{(3+\sqrt{3})^{2}}{2} a_{\mathrm{BN}}^{2}$ & $(1+\sqrt{2})^{2} a_{\mathrm{BN}}^{2}$
\end{tabular}


Таблица 2. Расчетные значения пьезоэлектрических и пироэлектрических коэффициентов 2D-наноаллотропов нитрида бора при $T=300 \mathrm{~K}$

\begin{tabular}{c|c|c|c|c}
\hline Параметр & $(\mathrm{BN})_{6}$ & $(\mathrm{BN})_{63(12)}$ & $(\mathrm{BN})_{664}$ & $(\mathrm{BN})_{44}$ \\
\hline$\alpha, \mathrm{N} / \mathrm{m}$ & 142 & 20.1 & 96.0 & 11.8 \\
$V_{2}, \mathrm{eV}$ & 11.7 & 6.9 & 4.4 & 142 \\
$V_{1}, \mathrm{eV}$ & 2.9 & 3.3 & 2.9 & 4.2 \\
$\gamma$ & 2.3 & 5.2 & 2.4 & 2.5 \\
$\alpha_{1}\left(T \gg \theta_{D}\right), 10^{-4}, \mathrm{~K}^{-1}$ & 0.21 & 8.4 & 15.6 & 1.0 \\
$\alpha_{1}(T=300 \mathrm{~K}), 10^{-4}, \mathrm{~K}^{-1}$ & 0.16 & 129 & 17.4 & \\
$e_{33}(T=300 \mathrm{~K}), 10^{-13} \mathrm{C} / \mathrm{m}$ & 1.19 & 69.6 & & 17.7 \\
$\gamma_{3}(T=300 \mathrm{~K}), 10^{-15} \mathrm{C} / \mathrm{m} \cdot \mathrm{K}$ & 0.28 & 16.5 & & 4.11
\end{tabular}

Константы центрального взаимодействия $\alpha$ взяты из работы [10].

чине к пироэлектрическим коэффициентам соответствующих фторографанов. Это обусловлено тем, что, согласно (7), (8), e 33 прямо пропорционально квадратному корню из температуры, а $\gamma_{3}$ обратно пропорционально ему же. Комнатная температура мала, чтобы создать такие же по величине дипольные моменты в элементарных ячейках 2D-нитридов бора, что и в ячейках фторографанов, но достаточна, чтобы привести к соизмеримому по величине пьезоэлектрическому эффекту в обоих классах наноаллотропов.

Заметим также, что для продольного пьезоэффекта в рассматриваемых наноструктурах величина пьезоэлектрического коэффициента $e_{22}=d_{22} c_{11}$, где $d_{22}-$ соответствующая компонента тензора пьезомодулей в матричной форме, а $c_{11}$ - компонента тензора упругих жесткостей, на три порядка выше, чем $e_{33}$. Например, для $(\mathrm{BN})_{6}: e_{22}=0.56 \cdot 10^{-12} \mathrm{C} / \mathrm{N} \cdot 405 \mathrm{~N} / \mathrm{m}$ $=2.27 \cdot 10^{-10} \mathrm{C} / \mathrm{m}[7]$.

Что касается продольного пироэлектрического эффекта, то, как отмечалось в п. 1, в рассматриваемых 2D-наноаллотропах нитрида бора он возможен лишь при наличии градиентов температуры в плоскости структуры.

Таким образом, для практического использования в различного рода датчиках для наноэлектромеханических систем управления у исследованных 2D-наноаллотропов нитрида бора могут представлять интерес их пироэлектрические свойства. При этом следует принимать во внимание их зависимость от температуры.

В заключении отметим, что приведенные в табл. 2 результаты для пьезоэлектрических коэффициентов $e_{33}$ носят оценочный характер, так как получены в предположении $T \ll \theta_{D}$, хотя в действительности имеет место случай $T \approx \theta_{D}$ При строгом подходе к задаче нужно учитывать также сложный характер элементарных ячеек рассматриваемых 2D-наноаллотропов, содержащих $N=2 Z$ атомов. В результате их фононный спектр будет содержать $2 N$ ветвей, из которых 2 будут акустическими, а остальные $2(N-1)$ - оптическими. Детальный расчет температурной зависимостей теплоемкостей таких 2D-наноаллотропов, представляет самостоятельный интерес. Возникающие при этом сложности не оправдывают включение подобных расчетов в данную работу, так как не повлияют существенным образом на порядок величины полученных результатов.

\section{Приложение}

Температура Дебая 2D-наноаллотропов может быть вычислена по формуле [14]

$$
\theta_{D}=\frac{\hbar}{k_{B}} \sqrt{8 \pi \frac{n_{2}}{N\left(\frac{1}{v_{L}^{2}}+\frac{1}{v_{T}^{2}}\right)}},
$$

где $n_{2}=N / S-$ поверхностная плотность атомов, которая может быть найдена как

$$
n_{2}=2 \frac{\rho_{2} N_{A}}{\mu_{\mathrm{B}}+\mu_{\mathrm{N}}}
$$

где $\rho_{2}$ - двумерная плотность материала наноаллотропа, $N_{A}-$ число Авогадро, $\mu_{\mathrm{B}}, \mu_{\mathrm{N}}-$ соответственно молярные массы бора и азота, а $N$ - общее число атомов в элементарной ячейке. Скорости продольных и поперечных упругих волн $v_{L}$ и $v_{T}$ соответственно для исследуемых наноаллотропов находятся из следующих выражений [15]:

$$
v_{L}=\sqrt{c_{11} / \rho_{2}}, \quad v_{T}=\sqrt{\left(c_{11}-c_{12}\right) /\left(2 \rho_{2}\right)}
$$

для $(\mathrm{BN})_{6},(\mathrm{BN})_{63(12)},(\mathrm{BN})_{664}$ и

$$
v_{L}=\sqrt{c_{11} / \rho_{2}}, \quad v_{T \max }=\sqrt{\left(c_{33}\right) /\left(\rho_{2}\right)}
$$

для $(\mathrm{BN}) 44$.

Подставляя (П2-П4) в (П1), получаем для температуры Дебая в соответствующих наноаллотропов следующие выражения:

$$
\begin{array}{r}
\theta_{D}=\frac{4 \hbar}{k_{B}} \sqrt{\frac{\pi N_{A}}{N\left(\mu_{\mathrm{B}}+\mu_{\mathrm{N}}\right)} \frac{c_{11}\left(c_{11}-c_{12}\right)}{3 c_{11}-c_{12}}}, \\
\theta_{D}=\frac{4 \hbar}{k_{B}} \sqrt{\frac{\pi N_{A}}{N\left(\mu_{\mathrm{B}}+\mu_{\mathrm{N}}\right)} \frac{c_{11} c_{33}}{c_{11}+c_{33}}} .
\end{array}
$$


Таблица 3. Значения температуры Дебая для 2D-наноаллотропов нитрида бора

\begin{tabular}{c|c|c|c|c}
\hline Параметр & $(\mathrm{BN})_{6}$ & $(\mathrm{BN})_{63(12)}$ & $(\mathrm{BN})_{664}$ & $(\mathrm{BN})_{44}$ \\
\hline$c_{11}, \mathrm{~N} / \mathrm{m}$ & 405 & 58.0 & 277 & 250 \\
$c_{12}, \mathrm{~N} / \mathrm{m}$ & 280 & 39.3 & 188 & 177 \\
$c_{33}, \mathrm{~N} / \mathrm{m}$ & - & - & - & 53.4 \\
$\theta_{D}, \mathrm{~K}$ & 1300 & 291 & 447 & 589
\end{tabular}

Значения $c_{11}, c_{12}, c_{33}$ взяты из работы [10].

Используя значение упругих жесткостей для исследуемых наноаллотропов из работы [12] и выражения (П5), (П6) можно рассчитать соответствующие для этих наноаллотропов значения температуры Дебая (см. табл. 3).

\section{Конфликт интересов}

Авторы заявляют, что у них нет конфликта интересов.

\section{Список литературы}

[1] S. Chandratre, P. Sharma, Appl. Phys. Lett. 100, 023114 (2012).

[2] M.T. Ong, E.J. Reed. FCS Nano 6, 1387 (2012).

[3] Р.А. Браже, А.И. Кочаев, А.А. Советкин. ФТТ 55, 1809 (2013).

[4] Р.А. Браже, А.И. Кочаев, А.А. Советкин. ФТТ 55, 1979 (2013).

[5] Р.А. Браже, Д.Е. Дулов. ФТТ 56, 2490 (2014).

[6] M. Droth, G. Burkard, V.M. Pereira. Phys. Rev. B 94, 075404 (2016).

[7] Р.А. Браже, Д.А. Долгов. ФТТ 61, (2019).

[8] P. Ares, T. Cea, M. Holwill, Y.B. Wang, R. Roldan, F. Guinea, D.V. Andreeva, L. Fumagalli, K.S. Novoselov, C. Woods. Adv. Matter. 32, (2020).

[9] Q. Wang, C.R. Bowen, R. Lewis, J. Chen, W. Lei, H. Zhang, M.-Y. Li, S. Jiang. Nano Energy 60, (2019).

[10] С.Ю. Давыдов. ФТТ 52, (2010).

[11] С.Ю. Давыдов. ФТП 47, (2013).

[12] Р.А. Браже, Д.А. Долгов. Изв. вузов. Электроника 25, (2020).

[13] С.Ю. Давыдов. Письма в ЖТФ 37, (2011).

[14] Р.А. Браже, В.С. Нефедов. ФТТ 54, (2012).

[15] Р.А. Браже, А.И. Кочаев, Р.М. Мефтахутдинов. ФТТ 53, (2011).

Редактор К.В. Емиев 